\title{
Scientific interactions in a virtual world
}

\author{
COVID-19 has forced a rethink of many practices we previously took for granted, and academic travel is \\ no exception. Virtual conferences have demonstrated their promise for encouraging a more equitable and \\ environmentally friendly future.
}

$\mathrm{T}$ he pandemic has brought about radical changes to academic life, including a transformation in the way scientists interact. In the pre-pandemic era, we took in-person conferences and meetings for granted. Although the shortcomings of in-person conferences-their inequality of access, time and financial costs, and environmental impacts-were becoming more and more visible, we couldn't imagine scientific meetings being anything other than primarily face-to-face affairs.

The pandemic has changed that: after initial cancellations, several conferences have moved to virtual platforms, with organizers seeking ways to recreate the in-person experience virtually. Although networking has become more challenging to achieve in a virtual format, virtual scientific conferences have thrived, with many planned as exclusively virtual events in 2021, despite the promise of an easing of COVID19 travel restrictions later in the year.

These changes to scientific interaction brought about by COVID-19 have given the academic community the opportunity to re-imagine what conferences in a post-pandemic era could look like. In a Comment published in this issue of Nature Human Behaviour, Sarabipour and colleagues do just that, offering recommendations on how scientific meetings can be improved to reduce their carbon footprint and increase their diversity, inclusivity, accessibility, early-career-researcher (ECR) participation, and networking and career-development opportunities.

As a starting point, Sarabipour and colleagues examined 270 in-person conferences across several disciplines that took place over 2 years. Among the several features the authors looked into, two particularly stand out: the environmental impact of in-person conferences and their exclusivity. The carbon footprint of a single audience member at a conference may equal what many people around the world produce in a year, whilst conference locations, attendance and travel fees render many in-person meetings affairs enjoyed only by a small elite. As the academic community considers the future of academic conferences, Sarabipour and colleagues identify several other areas for improvement for a hybrid or fully virtual conference future: codes of ethics and conduct, diversity and inclusion statements, richer opportunities for ECR training and networking, and much more.

Similarly to conferences, many departmental seminar series and journal clubs have seized the opportunity to cast the (virtual) net more widely, inviting speakers without constraints by travel arrangements and funds. This puts institutions across the globe on a more even footing with regard to who they may invite, serves as an equalizing force for scientists in remote locations who may now share their research with audiences in research hubs, and significantly reduces the need to travel and associated carbon-footprints.

Although in-person interactions will undoubtedly always have a place in academic life, returning to the pre-pandemic status quo for conferences, invited talks, and workshops is both undesirable and unlikely.

For us as editors, our work is primarily virtual: our team is based in two different locations (London and Berlin) and all our team meetings were virtual before the pandemic. The vast majority of our interactions with scientists are also virtualthrough email or phone or video calls-and this was also true before the pandemic. So, arguably, very little has changed in the nature of our work during COVID-19. However, before the pandemic we also each travelled to in-person conferences four to five times per year and did in-person lab visits. Our 'outreach' also contributed both to an increased carbon footprint and very limited access to universities in regions outside the locations of our offices or the conferences we attended, most of which took place in Northern Europe and North America.

The pandemic has meant that we too have been motivated to rethink how we interact with the scientific community and how we reach out to scientists beyond our physically accessible locations. Although we expect that some in-person conferences will continue to be a feature of our outreach, we are very keen to interact as much as we can with scientists from around the world and virtual media provide an inclusive and time-efficient opportunity to reach out to many more groups than previously. We will be delighted to hear from departments and groups, especially those located outside Northern Europe and North America, who would be interested in hosting us virtually for a talk on the publication process or on publishing as a career option for ECRs or to discuss their work and any issues of core interest to their discipline or scientific life. Please do reach out to us on humanbehaviour@nature.com and we will try to meet as many requests or invitations as is feasible for us.

In the midst of a harrowing pandemic, there may be a small silver lining for scientific interactions. It's up to us to sustain more environmentally friendly and inclusive modes of interaction not only now, but also in a post-pandemic era.

Published online: 19 March 2021 https://doi.org/10.1038/s41562-021-01091-y 